\title{
Review article \\ A review on the COVID-19, its history, diagnostic approaches, role of herbs and current world scenario
}

\author{
Devendra Singh ${ }^{1}$, Vishnu Agarwal ${ }^{1}$ \\ ${ }^{1}$ Department of Biotechnology, Motilal Nehru National Institute of Technology, Allahabad, 211 004, Uttar Pradesh, India
}

(Received: November 2020

Revised: May 2021

Accepted: May 2021)

Corresponding author: Vishnu Agarwal. Email: vishnua @mnnit.ac.in

\begin{abstract}
COVID- 19, a novel coronavirus disease caused by SARS-CoV-2 (severe acute respiratory syndrome caused by coronavirus-2) transmitted from person to person, is now declared as a pandemic worldwide by WHO. SARS-CoV2 belongs to the family of Coronaviridae. Covid-19 (Coronavirus disease 2019) is an infectious disease with clinical symptoms like fever, sore throat, sneezing, shortness of breath, fatigue, cough, diarrhoea, chills, and respiratory symptoms that has now entered into the new dangerous phase. This virus can easily extend and can cause severe illness to the global village. Till 7 Nov 2020, the total number of confirmed COVID-19 cases was 48534508, and the death number is 1231017, affecting, directly and indirectly, more than 215 territories worldwide. Hence an effective international strategy is required to control and prevent this disaster. To combat the coronavirus-2 different approaches have been initiated in scientific concern, but focussed attention should be given to the medical value of herbal plant extract to prevent these endemic type diseases. Hence, this review describes the symptoms and the role of herbal plants in treating the COVID-19.
\end{abstract}

Keywords: Lianhua-Qingwen; COVID-19; herbal plant; SARS-CoV-2.

\section{INTRODUCTION}

$\mathrm{F}$ rom December 2019, the novel coronavirus 2019 (COVID-19) disease, an unexpected pandemic caused by SARS-CoV-2 (severe acute respiratory syndrome caused by coronavirus-2), has caused severe panic to the global village. The coronavirus causes illness (alveolar injury as well as progressive respiratory stoppage), which finally leads to the death of the suffering person (1).

Developed and developing countries are continuously maximizing their efforts to control the spread of the virus and minimize the infection. Despite its similarity with the SAR-CoV virus, its diagnostic approaches and transmission efficiency are different, which is probably due to the change in nucleotide spike protein and its RBD (receptor binding domain) structure (2). To overcome this situation, a drug or a vaccine is urgently required to prevent mortality and morbidity. Many drugs are undergoing the clinical trial phase. Herbal medicinal plants are found promising in treating various diseases and may play a crucial role in defeating and treating this pandemic virus $(3,4)$.

\section{COVID-19 Overview}

Coronavirus is a fast-spreading virus capable of causing multiple system infection in animals, including $\mathrm{BCoV}$ (bovine respiratory Coronavirus) in cows, MHV (mouse hepatitis virus) in mouse, IBV (infectious bronchitis virus), also called as the avian virus in birds and chicken and severe respiratory infection such as MERS (Middle East respiratory syndrome) and SARS (severe acute respiratory syndrome) in humans $(5,6)$. The COVID-19 causes chronic and acute enteric, central nervous, as well as respiratory system infections in humans. Coronavirus is named due to the presence of crown-like spikes on its surfaces; enveloped, positive-stranded RNA virus belongs to the genus of the Coronaviridae family, and its genomic RNA size is $27-32 \mathrm{~kb}(6,7)$. This novel Coronavirus belongs to the $\beta$ Coronavirus, which is found to be close phylogenetically with bat SARS coronavirus exactly shares about $96.2 \%$ sequence similarity (8). Still, the exact origin of the COVID-19 virus remains a mystery to worldwide researchers, and researchers need to pinpoint the exact COVID-19 source (9). SARS-CoV-2 (severe acute respiratory syndrome caused by coronavirus-2) genomes have found mutated and can be divided into three types (Type A, Type B, and Type C). Type A and Type C major portions are found among Americans and Europeans. On the other side, Type B is mainly observed in East Asia (10). This virus in humans predominantly affects the upper respiratory tract with mild to severe illness (11).

\section{History}

In 1931, the first Coronavirus-based disease was diagnosed, while in 1965, from the humans first HCov-229E Coronavirus was isolated. However, SARS-CoV-1 (a member of sub-genus Sarbecovirus), which was realized as the often fatal respiratory infection, was firstly reported in 2002 in China with approximately $11 \%$ mortality (Fig. 1). The genome of SARS-CoV-1 is 29,727 nucleotides in length (12). Angiotensin-converting enzyme-2 (ACE-2) is a surface molecule present on the cells of the small intestinal epithelium and respiratory tract. This ACE2 is a receptor for SARS-CoV-1 and plays a vital role in protection from lung failure (13). Some common 
clinical symptoms of SARS-CoV-1 are fever, headache, cough, pneumonia, and dyspnea (11).

Later in 2012, in Saudi Arabia, MERS-CoV (Middle East respiratory syndrome coronavirus) causes endemic with a $34 \%$ mortality rate. It causes a viral respiratory infection (13). From infected camels, this virus is transmitted to humans (Fig. 1). Further, from 2012 to 2018, MERS-CoV cases were found in about 27 different countries, including Egypt and Qatar. The genome of MERS-CoV-1 is approximately 30,119 nucleotides in length, and DPP4 (dipeptidyl peptidase 4 ) is the receptor of MERS-CoV-1. DPP4 (multifunctional cell surface protein) protein is found on the epithelial cells of the kidney, respiratory tract, small intestine, liver and plays a crucial role in T cell activation (12). Some common symptoms of MERSOv-1 are sore throat, cough, abdominal pain, chest pain, fever, and vomiting (12).
In late 2019, COVID-19 (Coronavirus-19) infection case appears in China, which was caused by a coronavirus (SARS-CoV-2), which particularly affects the respiratory system of humans (Fig. 1). On 30 Jan, WHO declares COVID-19 as an international pandemic. On $11 \mathrm{Feb} 2020$, the WHO named the disease COVID-19 (9).

This newly discovered Coronavirus (SARS-CoV-2) encodes a glycosylated spike protein, which is mainly responsible for binding with the receptor, ACE-2 (14). The genetic material (RNA) of SARS-CoV-2 is enclosed by a lipid-bilayer envelope (15). Some common mode of transmission includes fecal-oral route, contact with an infected person, and via respiratory droplets (4).

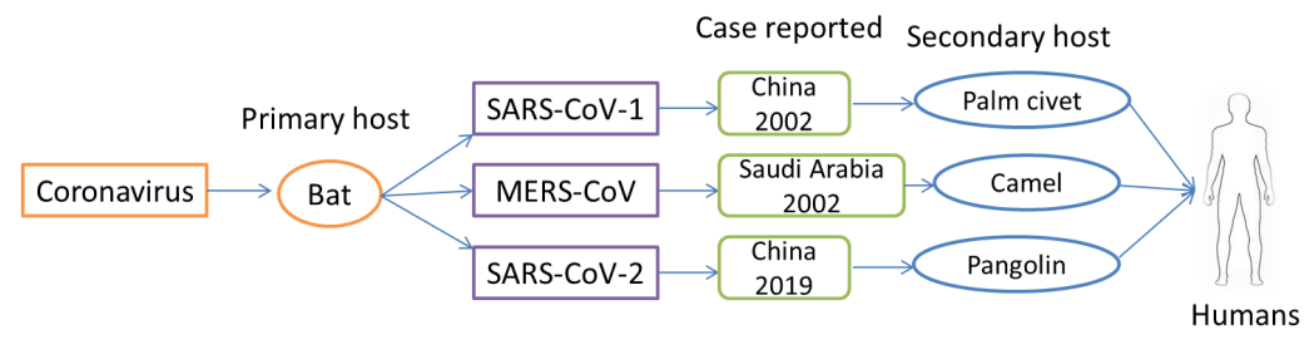

Fig. 1: Coronavirus transmission mode from host to humans

\section{Symptoms of COVID-19}

Coronavirus, a pandemic disease transmitted from one person to another via aerosol transmission, making it a threat for numerous individuals. Based on the collected information, the three common symptoms of COVID-19 are dry cough, fever, and difficulty in breathing, and other symptoms may include hypoxemia, a lesion in the lungs, tiredness, conjunctivitis, discoloration of toes or fingers, and diarrhea, as shown in figure 2 (4). These symptoms were approximate, observed within 5-6 days of infection (16).

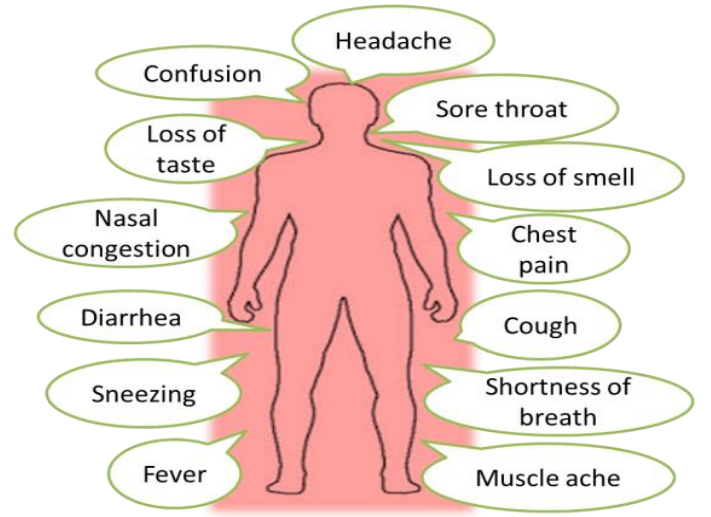

Fig. 2: Schematic representation of the COVID-19 symptoms

\section{Diagnostic method}

In this pandemic situation, the test is the only way to control the spreading of COVID-19. The COVID-19 virus is normally spread via the respiratory route through droplets from the infected patients. According to the CDC (centers for diseases control and prevention), two types of the diagnostic test for COVID-19 is available. a) Antibody tests, which are also known as the serological test, are mainly used to determine whether the person already had a COVID19 infection. In this test, a few drops of blood are taken from the individuals, and antibodies (IgM and $\operatorname{IgG}$ ) can be detected by using conventional ELISA tests. This antibody test is a rapid test that gives results within 20 minutes. The drawback related to this test is its poor specificity and lower sensitivity $(17,18)$.

b) Swab tests, Nasal (Nasopharyngeal) swab and throat (oropharyngeal) swab sample was collected from the patient and kept in viral medium (19). For higher sensitivity, both the nasal as well as throat swabs were combined together. Bronchoalveolar fluid, sputum, and faecal samples were also collected from the patients. The viral RNA is detected by using RT-PCR (reverse transcription-polymerase chain reaction). Many other antigen detection-based devices came into the market but had poor sensitivity (20).

\section{COVID-19 Management}

Some common FDA-approved drugs that are given to the hospital admitted COVID-19 patients include chloroquine, an antimalarial drug that inhibits the entry of Coronavirus. Studies have shown that the hydroxychloroquine, an analogue of chloroquine is 
more powerful and less toxic, can be used in place of chloroquine. In vitro condition, both the drug has effectively inhibited the SARS-CoV-2 activity (21). The clinical trial study performed in France and China provides evidence related to the effectiveness of both the drug (22). Other drug includes Ivermectin (antiparasitic drug), Camostat Mesylate (an anti-viral drug), Remdesivir (intravenous drug and found effective against MERS coronavirus), favipiravir (influenza drug), interferon-alpha (IFN- $\alpha$ ), arbidol and ribavirin $(23,24)$.

\section{Herbal plants}

The anti-viral property of herbal plants may play a crucial role in breaking the infection chain. Different in vitro and in vivo results have been reported about the activity of active components of herbal plants against the SAR-CoV-1 and influenza virus. But, only several clinical trial studies have been performed to analyze the effects of herbal plant extracts against the SAR-CoV-1 and influenza virus, and mostly clinical trial studies were focused on the herbal plant extract combinations or the TCM (traditional Chinese formulas) (25). Herbal plants and their active compounds are used for treating countlessly diseased and is the lead source for numerous drug discovery. Herbal plant-derived drugs such as thymol, an antifungal drug derived from Thymus vulgaris, artemisinin, an antimalarial drug derived from
Artemisia апnua, lovastatin, a hyperlipidemia drug synthesize from Aspergillus terreus, deserpidine, an anti-hypertensive drug derived from Rauwolfia canescens, veregen, a drug used for the treatment of genital/perianal warts was derived from the dried leaf of Camellia sinensis are the example of some FDA approved plant-derived drugs (25).

A research study confirm that glycyrrhizin, an active compound (derived from licorice roots), shows an anti-SARS-CoV effect by inhibiting the viral replication (26). Another active compound, glycyrrhizin, which is derived from the Glycyrrhiza glabra plant, also poses the anti-viral activity when tested in vitro against SARS-CoV, ten different clinical strains. The same results were also observed when baicalin, a compound derived from the Baikal skullcap plant, also displayed the anti-viral activity against SARS-CoV (27). It also inhibits the HIV-1 virus replication in vitro testing. Other herbal plants that pose the anti-viral activity against SARS coronavirus are the Eucalyptus tree, Japanese honeysuckle, and Korean ginseng, also known as Panax ginseng (27). The plant extracts of ginseng, garlic, ginger, eucalyptus, tea tree, Tianmingjing, Machixian, fish mint, Chinese mahogany, cape jasmine, and their active compounds exhibit anti-viral activity against the influenza virus (27; Table 1$)$.

Table1: Some promising plant-derived natural compounds with anti-coronavirus activity

\begin{tabular}{|c|c|c|c|}
\hline S.No. & Plant & Compound & Targeted coronavirus \\
\hline 1. & Glycyrrhiza glabra & Glycyrrhizin & SARS-Cov \\
\hline 2. & Selaginella tamariscina & Amentoflavoe & SARS-Cov \\
\hline 3. & Brassicaceae juncea & Sinigrin & SARS-Cov \\
\hline 4. & Maytenus imbricate & Tingenone & SARS-Cov \\
\hline 5. & Amaryllidaceae & Lycorine & SARS-Cov \\
\hline 6. & Tylophora indica & Tylophorine & TGEV \\
\hline 7. & Amorpha fruticose & Xanthoangelol & SARS-Cov \\
\hline
\end{tabular}

SARS-CoV represents Severe Acute Respiratory Syndrome caused by a coronavirus, and TGEV means Transmissible Gastroenteritis Virus.

\section{Chinese herbal drug}

Lianhua-Qingwen (a Chinese herbal formulation consists of 11 medicinal plants such as Armeniacae Semen Amarum, Forsythia Fructus, Ephedrae Herba, Dryopteris Crassirhizomatis Rhizoma, Lonicerae Japonicae Flos, Isatidis Radix, Glycyrrhizae Radix et Rhizoma, Houttuyniae Herba, Pogostemonis Herba, Rhodiolae Crenulatae Radix et Rhizoma and Rhei Radix \& Rhizoma along with a mineral medicine Gypsum Fibrosum and menthol) anti-inflammatory and inhibitory activity against SARS-CoV-2 was analyzed (28). This Chinese herbal formulation is mainly used to treat infections like influenza, fever, cough, bronchitis, sneezing, muscle ache, and fatigue.
In the USA, clinical testing on the Lianhua-Qingwen drug is in the process (29). The Chinese government has already recommended the Lianhua-Qingwen drug for the prevention and treatment of COVID-19 (30). The experimental results show that this herbal composition in a dose-dependent manner effectively inhibits the SARS-CoV-2 replication and also suppresses the pro-inflammatory cytokines release like IL- 6 and TNF- $\alpha$ (28). And in the case of COVID19 infections, an increase in cytokine was observed, so the obtained result will become more important.

\section{Current world scenario}

COVID-19 has adversely affected the health of humans throughout the world. At the time of preparing this manuscript on 7 Nov 2020, tabular data of the current world scenario was noted from the WHO dashboard (Table 2).

Table 2: Data showing the current scenario of some COVID-19 hotspot countries 


\begin{tabular}{|c|c|c|c|c|c|}
\hline S. No. & Country & Date of 1 $^{\text {st }}$ case & Total cases & Total Deaths & Transmission classification \\
\hline 1. & United States of America & 20 Jan 2020 & $9,387,978$ & 232,166 & Community transmission \\
\hline 2. & India & 30 Jan 2020 & $8,411,724$ & 124,985 & Clusters of cases \\
\hline 3. & Brazil & 25 Feb 2020 & $5,590,025$ & 161,106 & Community transmission \\
\hline 4. & Russian federation & 31 Jan 2020 & $1,733,440$ & 29,887 & Clusters of cases \\
\hline 5. & Globally & 17 Nov 2019 & $48,534,508$ & $1,231,017$ & - \\
\hline
\end{tabular}

\section{Preventive measures}

Till date, there is no clinically proven treatment drug are available in the market for COVID suffering patients, so participation in the gathering should be avoided. According to WHO and ICMR guidelines, social distancing, avoiding the gathering, home isolation, and home quarantine if any symptoms were visualized (Fig. 3).

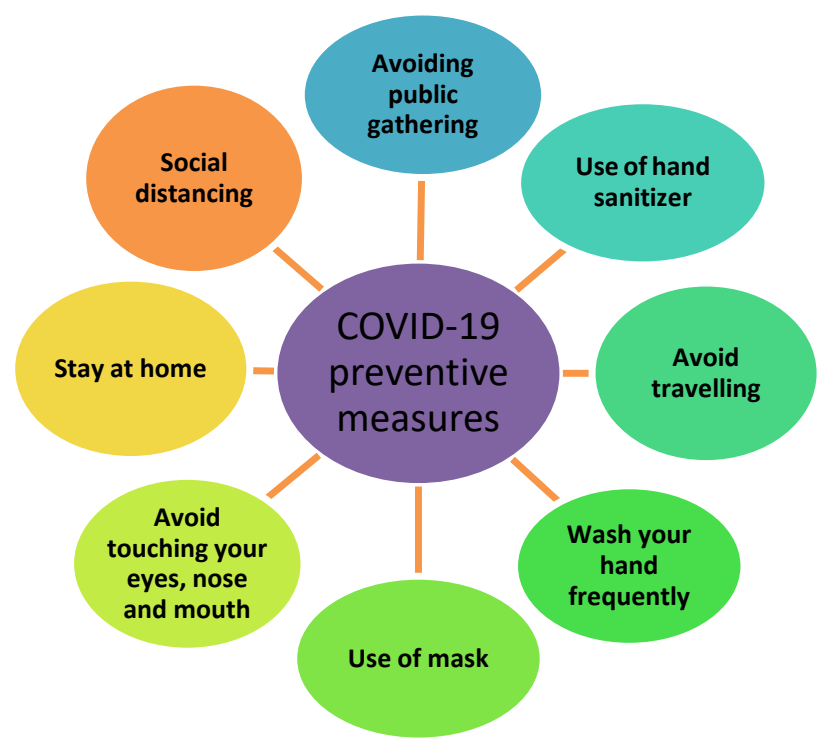

Fig. 3: Illustrative representation of nCOVID-19 safety measures

\section{CONCLUSION}

The recent outbreak of COVID-19 has now become a global threat to humanity. In this review, the information regarding the COVID-19, history, its symptoms, diagnostic method, management of COVID-19, and current world scenario has been summarized. As of now, no drug for the treatment of COVID-19 has been available for patients, and only a few numbers of allopathic drugs are found effective and are given to hospitalized patients. This review provides information and obvious evidence regarding the use of anti-viral herbal plant and their compounds as a preventive agent against SARS-CoV-2. These herbal plants in single or in combination can be served as an alternative preventive therapy against SARSCoV-2. However, this hypothesis should be experimentally validated via SARS-Cov-2 infection models and clinically tested on COVID-19 patients.

\section{ACKNOWLEDGEMENT}

We would like to acknowledge TEQIP for the financial assistantship.

\section{CONFLICT OF INTEREST}

No conflict of interest exists between the authors.

\section{REFERENCES}

1. Qiao, J. What are the risks of COVID-19 infection in pregnant women? Lancet. 2020; 395: 760-762.

2. Xu, Z., Shi, L., Wang, Y., Zhang, J., Huang, L., Zhang, C., et al., Pathological findings of COVID-19 associated with acute respiratory distress syndrome. Lancet. Respire. Med. 2020; 8: 420-422.

3. Wan, Y., Shang, J., Graham, R., Baric, R. S., Li, F. Receptor recognition by novel coronavirus from Wuhan: an analysis based on decade-long structural studies of SARS. Journal of Virology. 2020; 94: e00127-20.

4. Balachandar, V., Mahalaxmi, I., Kaavya, J., Vivekanandhan, G., Ajithkumar, S., Arul, N., et al., COVID-19: emerging protective measures. European Review for Medical and Pharmacological Sciences. 2020; 24: 3422-3425.

5. Jiang, S., Shi, Z., Shu, Y., Song, J., Gao, G. F., Tan, W., et al., A distinct name is needed for the new coronavirus. Lancet. 2020; 395(10228): 949.

6. Weiss, S. R., Navas-Martin, S. Coronavirus pathogenesis and the emerging pathogen severe acute respiratory syndrome coronavirus. Microbiology and Molecular Biology Reviews. 2005; 69: 635-664.

7. Neuman, B. W., Adair, B. D., Yoshioka, C., Quispe, J. D., Orca, G., Kuhn, P., et al., Supramolecular architecture of severe acute respiratory syndrome coronavirus revealed by electron cryomicroscopy. Journal of Virology. 2006; 80: 7918-7928.

8. Chan, J. F. W., Kok, K. H., Zhu, Z., Chu, H., To, K. K. W., Yuan, S., et al., Genomic characterization of the 2019 novel human-pathogenic coronavirus isolated from a patient with atypical pneumonia after visiting Wuhan. Emerging Microbes and Infections. 2020; 9: 221-236.

9. Guarner, J. Three emerging coronaviruses in two decades. American Journal of Clinical Pathology. 2020; 153: 420-421.

10. Forster, P., Forster, L., Renfrew, C., Forster, M. Phylogenetic network analysis of SARS-CoV-2 genomes. Proceedings of the National Academy of Sciences of the United States of America. 2020; 117: 9241-9243.

11. Huang, C., Wang, Y., Li, X., Ren, L., Zhao, J., Hu, Y., et al., Clinical features of patients infected with 2019 novel coronavirus in Wuhan, China. Lancet. 2020; 395: 497-506.

12. Song, Z., Xu, Y., Bao, L., Zhang, L., Yu, P., Qu, Y., et al., From SARS to MERS, thrusting coronaviruses into the spotlight. Viruses. 2019; 11: 59.

13. Yin, Y., Wunderink, R. G. MERS, SARS and other coronaviruses as causes of pneumonia. Respirology. 2018; 23: 130-137.

14. Bosch, B. J., van der Zee, R., de Haan, C. A., Rottier, P. $\mathrm{J}$. The coronavirus spike protein is a class I virus fusion protein: structural and functional characterization of the fusion core complex. Journal of Virology. 2003; 77: 8801-8811.

15. Siu, Y. L., Teoh, K. T., Lo, J., Chan, C. M., Kien, F., Escriou, N., et al., Structural proteins of the severe acute respiratory syndrome coronavirus are required for efficient assembly, trafficking, and release of virus-like particles. Journal of Virology. 2008; 82: 11318-11330.

16. Menni, C., Valdes, A., Freydin, M. B., Ganesh, S., Moustafa, J. E., Visconti, A., et al., Loss of smell and 
taste in combination with other symptoms is a strong predictor of COVID-19 infection. MedRxiv. 2020.

17. Li, Z., Yi, Y., Luo, X., Xiong, N., Liu, Y., Li, S., et al., Development and clinical application of a rapid IgMIgG combined antibody test for SARS-CoV-2 infection diagnosis. Journal of Medical Virology. 2020; 92(9): $1518-1524$.

18. Zhang, W., Du, R. H., Li, B., Zheng, X. S., Yang, X. L., $\mathrm{Hu}$, B., et al., Molecular and serological investigation of 2019-nCoV infected patients: implication of multiple shedding routes. Emerging Microbes and Infections. 2020; 9: 386-389.

19. Carver, C., Jones, N. Comparative accuracy of oropharyngeal and nasopharyngeal swabs for diagnosis of COVID-19. Oxford COVID-19 evidence service team centre for evidence based medicine. 2020.

20. Sheridan, C. Fast, portable tests come online to curb coronavirus pandemic. Nature Biotechnology. 2020; 38: 509-511.

21. Yao, X., Ye, F., Zhang, M., Cui, C., Huang, B., Niu, P., et al., In vitro antiviral activity and projection of optimized dosing design of hydroxychloroquine for the treatment of severe acute respiratory syndrome coronavirus 2 (SARS- CoV-2). Clinical Infectious Diseases. 2020; 71: 732-739.

22. Gao, J., Tian, Z., Yang, X. Breakthrough: chloroquine phosphate has shown apparent efficacy in treatment of COVID-19 associated pneumonia in clinical studies. BioScience Trends. 2020; 14: 72-73.

23. Wagstaff, K. M., Sivakumaran, H., Heaton, S. M., Harrich, D., Jans, D. A. Ivermectin is a specific inhibitor of importin $\alpha / \beta$-mediated nuclear import able to inhibit replication of HIV-1 and dengue virus. Biochemical Journal. 2012; 443: 851-856.

24. Furuta, Y., Takahashi, K., Shiraki, K., Sakamoto, K., Smee, D. F., Barnard, D. L., et al., T-705 (favipiravir) and related compounds: novel broad- spectrum inhibitors of RNA viral infections. Antiviral Research. 2009; 82: 95-102.

25. Luo, H., Tang, Q. L., Shang, Y. X., Liang, S. B., Yang, M., Robinson, N., et al., Can Chinese medicine be used for prevention of Corona Virus Disease 2019 (COVID19)? A review of historical classics, research evidence and current prevention programs. Chinese Journal of Integrative Medicine. 2020; 26: 243-250.

26. Hoever, G., Baltina, L., Michaelis, M., Kondratenko, R., Baltina, L., Tolstikov, G. A., et al., Antiviral activity of glycyrrhizic acid derivatives against SARScoronavirus. Journal of Medicinal Chemistry. 2005; 48: 1256-1259.

27. Chen, F., Chan, K. H., Jiang, Y., Kao, R. Y. T., Lu, H. T., Fan, K. W., et al., In vitro susceptibility of 10 clinical isolates of SARS coronavirus to selected anti-viral compounds. Journal of Clinical Virology. 2004; 31: 6975.

28. Ding, Y., Zeng, L., Li, R., Chen, Q., Zhou, B., Chen, Q., et al., The Chinese prescription lianhuaqingwen capsule exerts anti-influenza activity through the inhibition of viral propagation and impacts immune function. BMC Complementary and Alternative Medicine 2017; 17 : 130.

29. Gao, D., Niu, M., Wei, S. Z., Zhang, C. E., Zhou, Y. F., Yang, Z. W., et al., Identification of a pharmacological biomarker for the bioassay-based quality control of a thirteen-component TCM formula (Lianhua Qingwen) used in treating influenza A virus (H1N1) infection. Frontiers in Pharmacology. 2020; 11: 746.

30. Yang, Y., Islam, M. S., Wang, J., Li, Y., Chen, X. Traditional Chinese medicine in the treatment of patients infected with 2019-New Coronavirus (SARS-CoV-2): A Review and Perspective. International Journal of Biological Sciences. 2020; 16: 1708-1717. 